\title{
Performance Improvement for Medical Image Transmission Systems using Turbo-Trellis Coded Modulation (TTCM)
}

\author{
Menezla Fayssal \\ Department of Technology and Science, Nour Bachir \\ University Center of El-Bayadh, Algeria and \\ LEPO Laboratory, Djillali Liabès University, Algeria \\ menezla@yahoo.fr
}

Mahdjoub Zoubir

LEPO Laboratory

Djillali Liabès University

Sidi Bel-Abbès. Algeria

z.mahdjoub@yahoo.fr

\author{
Bendelhoum Mohammed Sofiane \\ Department of Technology and Science \\ Nour Bachir University Center of El-BayadhEl-Bayadh \\ El-Bayadh, Algeria \\ Bendelhoum_med@yahoo.fr \\ Mohamed Reda Lahcene \\ Department of Technology and Science \\ Nour Bachir University Center of El-BayadhEl-Bayadh \\ El-Bayadh, Algeria \\ lahcenereda1@gmail.com
}

\author{
Abdelhalim Zerroug \\ Sonatrach Oil and Gas Company \\ Algeria \\ eloual@yahoo.fr
}

\begin{abstract}
Digital images have become an essential working tool in several areas such as the medical field, the satellite and astronomical field, film production, etc. The efficiency of a transmission system to exchange digital images is crucial to allow better and accurate reception. Generally, transmitted images are infected with noise. In the medical field, this noise makes the process of diagnosing difficult. To eliminate the transmission errors, an Error Correcting Code (ECC) can be used with the aim to guarantee excellent reception of the images and allowing a good diagnosis. In this paper, source and channel encoding/ decoding functions are studied during medical image transmission (LUNG). At first, the Turbo-Code (TC) is used as ECC and subsequently the Turbo-Trellis Coded Modulation (TTCM). The simulation results represent the curves giving the Bit Error Rate (BER) as a function of the signal to noise ratio $\left(E_{b} / N_{0}\right)$. In order to compare these two systems properly, the MSSIM (Mean Structural Similarity) parameter was used. The obtained results showed the effect and importance of ECC on the transmission of medical images using TTCM which gave better results compared to $\mathrm{TC}$ with regard to increasing the performance of the transmission system by eliminating transmission noise.
\end{abstract}

Keywords-channel coding; turbo-codes; TTCM; MSSIM; medical image

\section{INTRODUCTION}

Given their importance and their massive use, digital images have become an essential mean of communication.
Medical images are widely used as a diagnostic tool and in biomedical research [1]. Sometimes it is necessary to transmit such images to distant doctors to have their opinions/diagnosis. The transmitted images are very sensitive to transmission errors, so it is very important to protect them against transmission noise in order to have on reception a sharp and clear image identical to the original one, allowing doctors to make a good diagnosis. As a solution to the noise problem, Error Correcting Code (ECC) can be added to the medical image transmission chain in order to eliminate the effect of noise during transmission. Such codes are: block codes, convolutional codes, LDPC codes [2], Turbo-Code (TC) and Turbo-Trellis Coded Modulation (TTCM). TC and ECC allow approaching the theoretical limit of correction predicted by Shannon more than 60 years ago [3-5]. More recently, a new family of ECCs, the TTCM [6,7], was introduced.

In this article, we first explain the basic principles of TC and TTCM, then we will propose the model of our digital image transmission chain, in which we will transmit a medical image of a lung, using TC and TTCM in order to make a comparison between the two systems and propose the best solution for noisy transmissions. To evaluate our simulation results, additively to BER and signal to noise ratio $\left(E_{b} / N_{0}\right)$ parameters, we will use the MSSIM (Mean Structural Similarity) parameter to see and analyze the results in a subjective and objective way. The image [8] used in this article shows infected lungs with the Corona virus.

Corresponding author: Menezla Fayssal 


\section{TURBO-CODE}

The TC corrects error produced by transmission noise. This code, invented and presented by Claude-Berrou [4], is obtained by concatenation of two or more ECCs of low complexity [5], allowing to approach the theoretical limit of correction. Their decoding uses an iterative (or turbo) process. In the principal plan of TC [9] the input binary message of length $k$ is encoded in its natural order and in a permuted order, by two coders called $C_{1}$ and $C_{2}$. The two elementary coders are identical, but this is not necessary. In our example, the performance of the natural encoding, without punching is $1 / 3$, for each source bit $\left(d_{k}\right)$ three bits $\left(x, y_{1}, y_{2}\right)$ are sent on the channel [7]. Turbodecoding is carried out according to the principle of iterative decoding [10], based on the use of SISO (Soft-Input, SoftOutput) decoders which exchange information from each other's reliability $Z_{k}[11]$. These are called extrinsic information, through a feedback, to improve the correction over the iterations. The circuit of a turbo-decoder is constituted by cascading $P$ modules, corresponding to the $P$ identical decoding iterations and its structure is perfectly modular [9]. The input of the $P^{\text {th }}$ module consists of the properly received delayed sequences $\left\{x_{k}^{\prime}\right\}_{P-1},\left\{y_{k}^{\prime}\right\}_{P-1}$ and the sequence $\left\{Z_{k}^{\prime}\right\}_{P-1}$ of the feedback generated by the $(P-1)^{\text {th }}$ module.

\section{TURBO-TRELLIS CODED MODULATION}

TTCM is an ECC introduced by Robertson and Wörz in $1995[12,13]$. This code is based on the concatenation of two Trellis Coded Modulations (TCM). The TCM was introduced in the early ' $80 \mathrm{~s}$ [14] and is based on the joint optimization of error correcting coding and modulation. Coding is done directly in the signal space, so the ECC and the binary signal code of the modulation can be represented all along using a single trellis. The optimization criterion for a TCM consists in maximizing the minimum Euclidean distance between two coded sequences. In the TTCM scheme each MCT coder consists of a recursive systematic convolutional coder, or CSR coder, of efficiency $q /(q+1)$ and of a modulation without order memory $Q=2^{q+1}$ [15]. Binary symbols from the source are grouped by $q$-bit symbols. These symbols are coded by the first MCT in the order they are issued by the source and by the second after interleaving.

\section{SimUlation RESUltS}

To eliminate the noise introduced by the channel and to improve the reception quality of a given communication system, an error correction coder must be used [9, 16-20]. The aim of the conducted simulation is to show the effect of the TC and TTCM on a medical image transmission chain in a Gaussian canal and to make a comparison between the performance of these two codes. In all simulations we use code of 1/2 return and MAQ16 modulation to have the same spectral efficiency in the two transmission chains. The spectral efficiency of a transmission is given by $[9,16]$ :

$$
\eta=R \log _{2} M \quad(\mathrm{bit} / \mathrm{s} / \mathrm{Hz})
$$

So in both cases of our transmission chain, spectral efficiency is given by:

$$
\eta=1 / 2 \log _{2} 16=2 \mathrm{bit} / \mathrm{s} / \mathrm{Hz}
$$

The results are obtained by evaluating the signal to noise ratio $\left(\mathrm{E}_{\mathrm{b}} / \mathrm{N}_{0}\right)$ for a number of iterations. In each case the (BER) and the Structural Similarity Index (SSIM) are calculated. Two ways are generally used to measure the quality of degraded images, the subjective methods and the objective methods. In this paper, besides the evaluation criteria, we will use a new criterion, which is the SSIM. This criterion presents the similarity and compares the luminosity, the contrast, and the structure between each pair of vectors of the two images (original and received). The SSIM between two signals $x$ and $y$ is given by [9]:

$$
\operatorname{SSIM}(x, y)=\mathrm{L}(x, y), \mathrm{C}(x, y) \text { and } \mathrm{S}(x, y)
$$

where $\mathrm{L}(x, y)$ represents the comparison of the brightness between the original and the received image, $\mathrm{C}(x, y)$ represents the comparison of the contrast between the original and the received image, and $\mathrm{S}(x, y)$ represents the comparison of the structure between the original image and the received image.

The proposed simulation algorithm for the image transmission chain coded with TC and TTCM is presented in Figure 1.

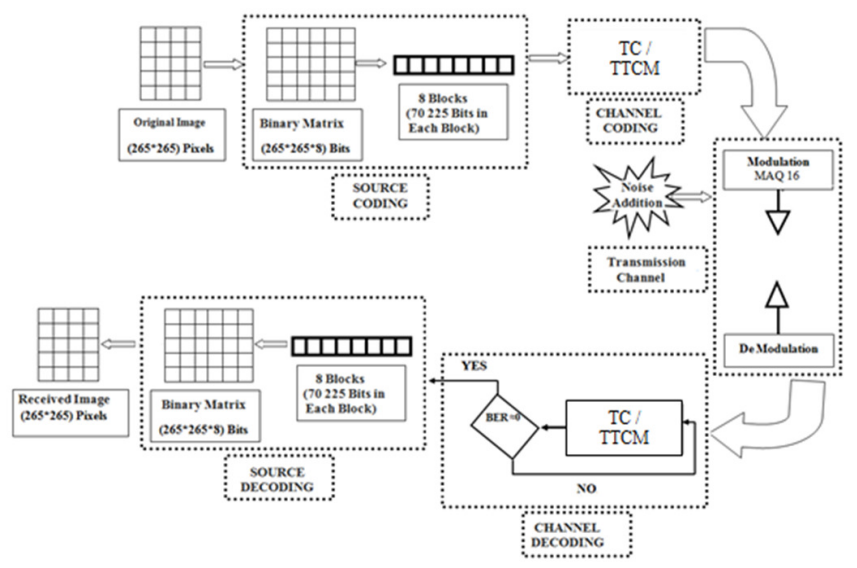

Fig. 1. Block diagram of the image transmission chain.

Figure 2 shows the effect of TC in the transmission chain of the image. The obtained simulation results represent the evaluation of the BER function of the signal-to-noise ratio in a image transmission in a Gaussian channel using TC with rate $R=1 / 2$ and MAQ16 modulation.

Figure 3 shows the effect of TTCM in the transmission chain. The obtained simulation results represent the evaluation of the BER function of the signal-to-noise ratio in a medical image transmission in a Gaussian Channel using TTCM with rate $R=1 / 2$ and MAQ16 modulation.

Figure 4 shows the performance comparison between the TC whit $y=2$ and TTCM whit $\eta=2$ in the transmission chain. 


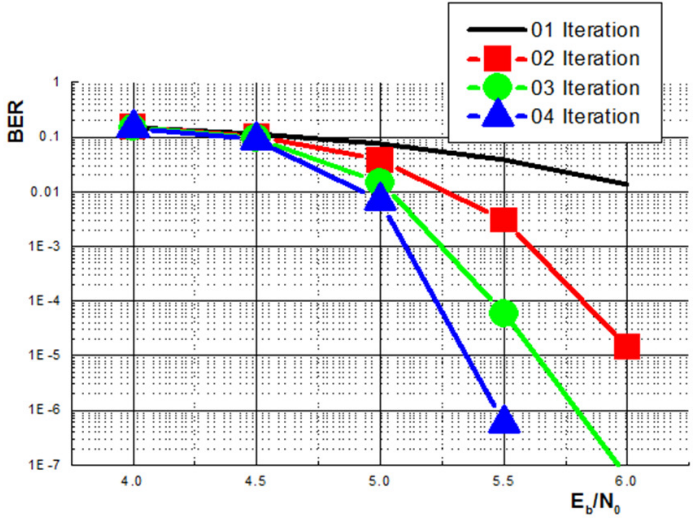

Fig. 2. The influence of TC on the transmission of a medical image in a Gaussian channel with $\eta=2 \mathrm{bit} / \mathrm{s} / \mathrm{Hz}$.

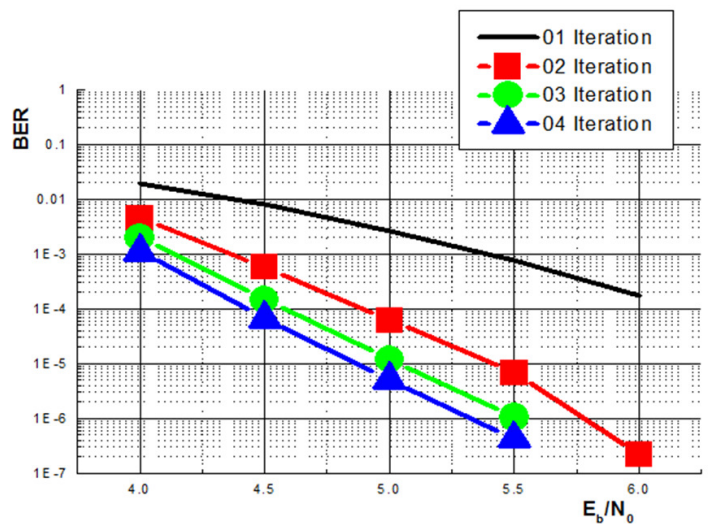

Fig. 3. The influence of TTCM on the transmission of a medical image in a Gaussian channel with $\eta=2 \mathrm{bit} / \mathrm{s} / \mathrm{Hz}$.

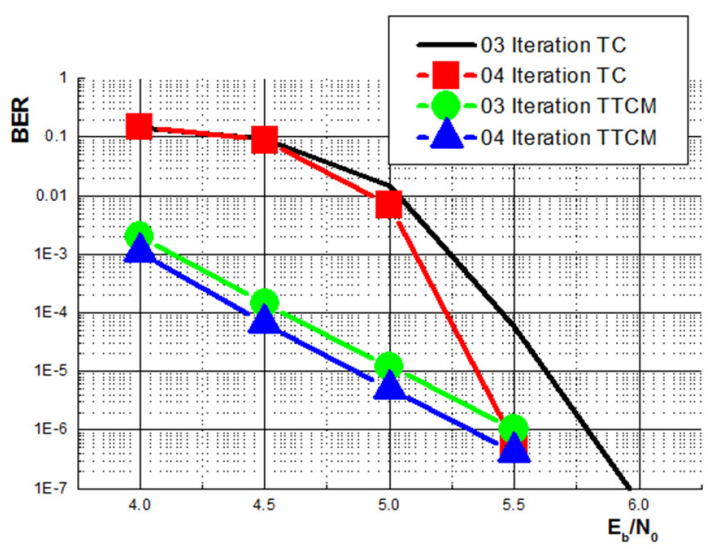

Fig. 4. Comparison between the TC and TTCM on the transmission of a medical image in a Gaussian channel with $y=2 \mathrm{bit} / \mathrm{s} / \mathrm{Hz}$.

The BER measurement gives a numerical value on the damage, but it does not describe its type, it does not quite represent the quality perceived by human observers. For medical imaging applications, where the degraded images must eventually be examined by experts, traditional evaluation remains insufficient. For this reason, objective approaches are needed to assess the medical imaging quality [21]. A new paradigm is evaluated to estimate the quality of medical images. For this reason and to have more details on our system the SSIM will be used as indicated above. Figures 5 and 6 show the effect of TC and TTCM in the transmission chain of a medical image. The obtained simulation results represent the evaluation of the SSIM function of the signal-to-noise ratio in a medical image transmission in a Gaussian channel using TC and TTCM with $R=1 / 2$ rate and MAQ16 modulation. Figure 7 shows the comparison between the performance of the TC and TTCM. The results are represented in detail in Tables I and II and the histograms in Figures 8 and 9.

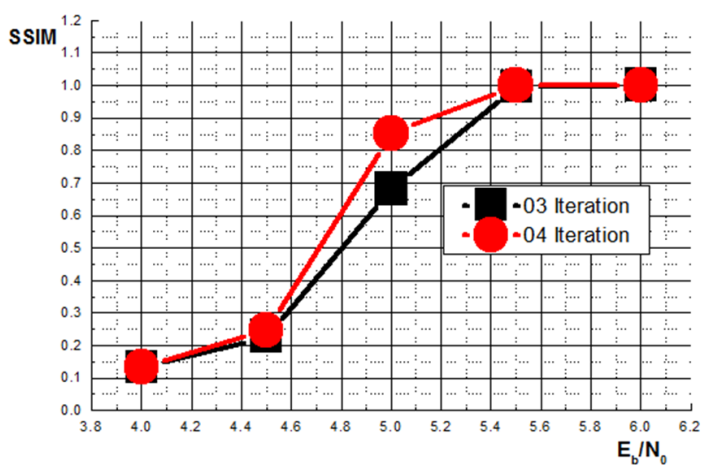

Fig. 5. The obtained SSIM using TC on the transmission of a medical image in a Gaussian channel with $\eta=2 \mathrm{bit} / \mathrm{s} / \mathrm{Hz}$.

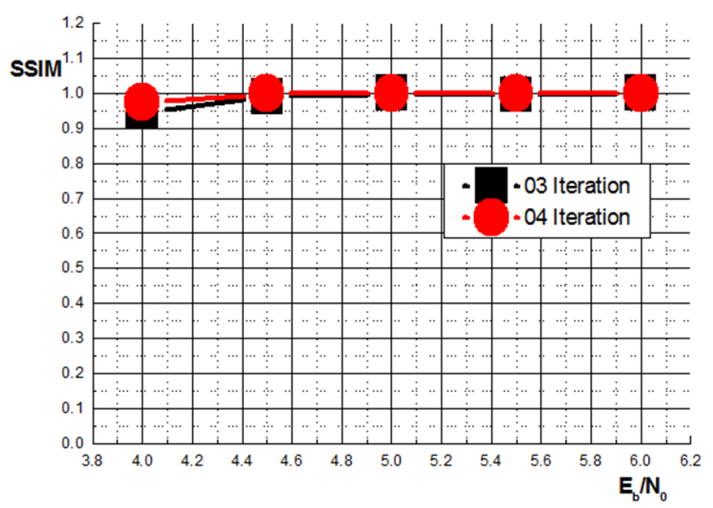

Fig. 6. The obtained SSIM using TTCM on the transmission of a medical image in a Gaussian channel with $\eta=2 \mathrm{bit} / \mathrm{s} / \mathrm{Hz}$.

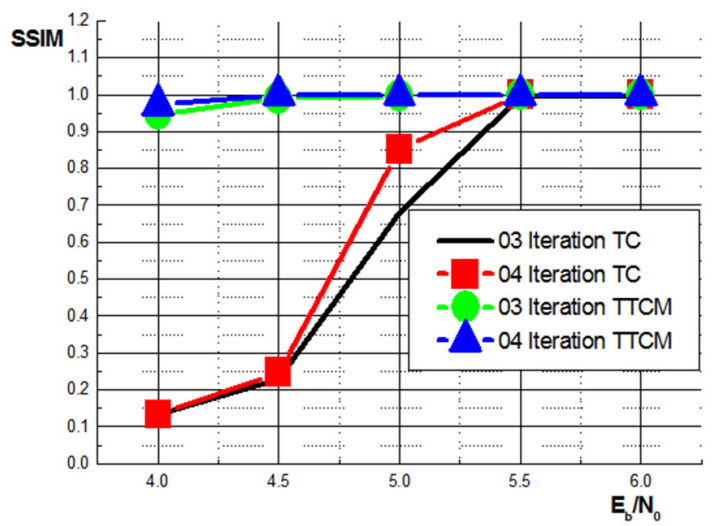

Fig. 7. Comparison of the SSIM between the TC and the TTCM on the transmission of a medical image in the Gaussian channel with $\eta=2 \mathrm{bit} / \mathrm{s} / \mathrm{Hz}$. 
TABLE I. OBTAINED RESULTS OF BER AS A FUNCTION OF THE SIGNAL TO NOISE RATIO AND THE NUMBER OF ITERATIONS

\begin{tabular}{|c|c|c|c|}
\hline \multirow{2}{*}{$\begin{array}{l}E_{b} / N_{0} \\
(\mathrm{~dB})\end{array}$} & \multirow{2}{*}{ Iteration No. } & \multicolumn{2}{|r|}{ BER } \\
\hline & & TC & TTCM \\
\hline \multirow{4}{*}{4} & 1 & 0.150801 & 0.0196 \\
\hline & 2 & 0.1494215 & 0.0046 \\
\hline & 3 & 0.14854397 & 0.0020 \\
\hline & 4 & 0.148309 & 0.0011 \\
\hline \multirow{4}{*}{4.5} & 1 & 0.11651121 & 0.00797721609113564 \\
\hline & 2 & 0.10195265 & 0.000585083659665361 \\
\hline & 3 & 0.0946173 & 0.000145781416874333 \\
\hline & 4 & 0.08811321 & $6.62157351370595 \mathrm{e}^{-05}$ \\
\hline \multirow{4}{*}{5} & 1 & 0.07558384 & 0.002655927376290500 \\
\hline & 2 & 0.03763973 & $6.20327518689925 \mathrm{e}^{-05}$ \\
\hline & 3 & 0.01492702 & $1.19971520113920 \mathrm{e}^{-05}$ \\
\hline & 4 & 0.006852973 & $5.00177999288003 \mathrm{e}^{-06}$ \\
\hline \multirow{4}{*}{5.5} & 1 & 0.038408685 & 0.000749875400498398 \\
\hline & 2 & 0.002993948 & $6.63937344250623 \mathrm{e}^{-06}$ \\
\hline & 3 & 0.000058739766 & $1.03239587041652 \mathrm{e}^{-06}$ \\
\hline & 4 & 0. & $4.22997508009968 \mathrm{e}^{-07}$ \\
\hline \multirow{4}{*}{6} & 1 & 0.013770025 & 0.000176913492346031 \\
\hline & 2 & 0.000014239943 & $2.13599145603418 \mathrm{e}^{-07}$ \\
\hline & 3 & 0 . & 0 . \\
\hline & 4 & 0. & 0 . \\
\hline
\end{tabular}

घ 1 Iteration $\quad \mathbf{2}$ Iteration $\quad \mathbf{3}$ Iteration $\quad \mathbf{4}$ Iteration

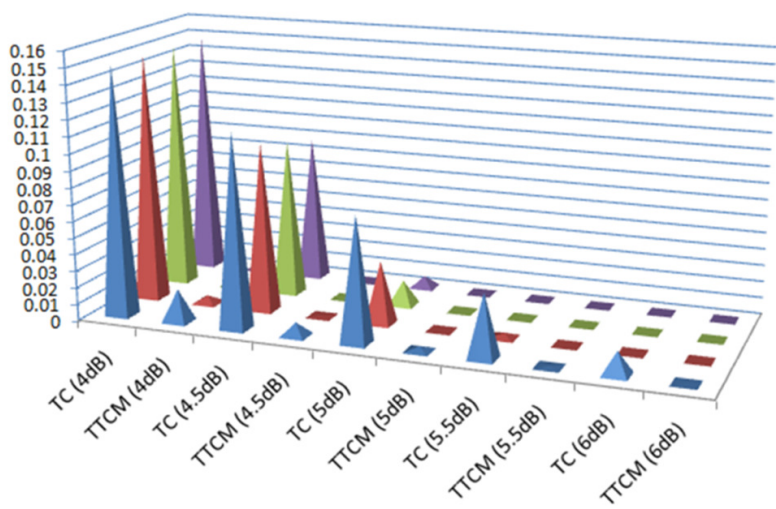

Fig. 8. Graphical representation of the data in Table I.

\section{COMMENTS AND INTERPRETATION OF THE OBTAINED RESULTS}

From the simulation results, it can be noticed that the role of the ECCs is very important in the transmission of images. The simulation results show that by using transmission systems with TTCM, there is a coding gain compared to transmission systems that use TC. With TTCM, the BER and SSIM results as functions of SNR are quite satisfactory. In the case of TTCM it is more adequate to remain at the 3rd iteration, allowing the receiver to have an easy structure to put in. The decoding will be simpler and the time shorter leading to optimal results. To see the importance of our work and in order to justify the reliability of our system, we have compared the obtained results with the ones obtained in $[21,22]$. In these articles, the authors chose joint encoding with TCM with a spectral efficiency of $\eta=2 \mathrm{bit} / \mathrm{s} / \mathrm{Hz}$. It can be said that the obtained results of the current study have a significant coding gain and also we arrived at SSIM $=1(100 \%)$ which is not the case in the cited articles.
TABLE II. OBTAINED RESULTS OF SSIM AS A FUNCTION OF THE SIGNAL TO NOISE RATIO AND THE NUMBER OF ITERATIONS

\begin{tabular}{|c|c|c|c|}
\hline \multirow{2}{*}{$\begin{array}{l}E_{b} / N_{0} \\
\text { (dB) }\end{array}$} & \multirow{2}{*}{ Iteration No. } & \multicolumn{2}{|c|}{ SSIM } \\
\hline & & TC & TTCM \\
\hline \multirow{3}{*}{4} & 3 & 0.132 & $\begin{array}{l}0.947 \\
9477^{\circ}\end{array}$ \\
\hline & \multirow{2}{*}{4} & 0.133 & 0.973 \\
\hline & & $13.3 \%$ & $97.3 \%$ \\
\hline \multirow{4}{*}{4.5} & \multirow{2}{*}{3} & 0.227 & 0.989 \\
\hline & & $22.7 \%$ & $98.9 \%$ \\
\hline & \multirow[b]{2}{*}{4} & 0.247 & 0.999 \\
\hline & & $24.7 \%$ & $99.9 \%$ \\
\hline \multirow{4}{*}{5} & \multirow{2}{*}{3} & 0.681 & 0.996 \\
\hline & & $68.1 \%$ & $99.6 \%$ \\
\hline & \multirow{2}{*}{4} & 0.852 & 1 \\
\hline & & $85.2 \%$ & $100 \%$ \\
\hline \multirow{4}{*}{5.5} & \multirow{2}{*}{3} & 0.995 & 1 \\
\hline & & $99.5 \%$ & $100 \%$ \\
\hline & \multirow{2}{*}{4} & 1 & 1 \\
\hline & & $100 \%$ & $100 \%$ \\
\hline \multirow{3}{*}{6} & \multirow{2}{*}{3} & 1 & 1 \\
\hline & & $100 \%$ & $100 \%$ \\
\hline & 4 & $\begin{array}{c}1 \\
1\end{array}$ & $\begin{array}{c}1 \\
100 \%\end{array}$ \\
\hline
\end{tabular}

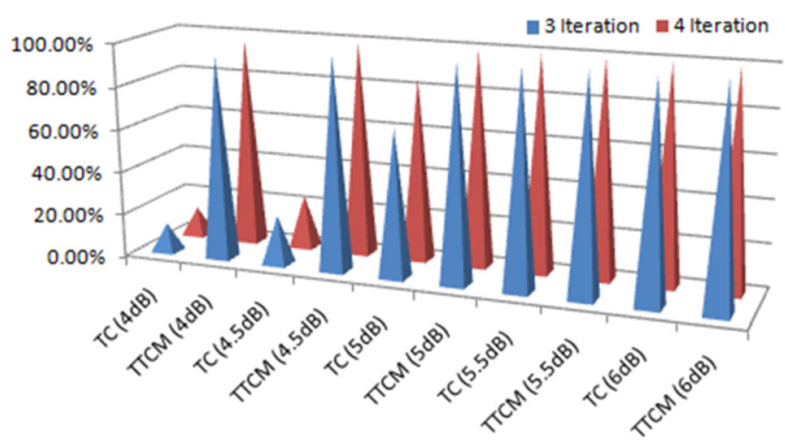

Fig. 9. Graphical representation of the data in Table II.

\section{CONCLUSION}

The main objective of our work is to study the performance of TC and TTCM when transmitting medical images over a Gauss channel. The simulation results obviously depict that our proposed transmission scheme performs better for the transmission of digital images and BER is significantly decreased $(\mathrm{BER}=0)$. This efficiency leads to the best reception quality of the digital images, $100 \%$ close to the transmitted ones (SSIM=1). Moreover, we demonstrated that the TTCM implementation outperforms the TC. By using TTCM, it is suggested to stop the process at the $3 \mathrm{rd}$ iteration. This allows having a simple architecture of the decoder and optimizes the decoding process in terms of time and complexity. The obtained results make the proposed transmission scheme a strong candidate when transmitting medical images, allowing medical staff to have best quality at the reception and facilitate the diagnosis process. The obtained results are better than the results found in [21].

\section{ACKNOWLEDGEMENT}

We express our sincere thanks to the General Directorate of Scientific Research and Technological Development (DGRSDT) for their support in the development of this work. 


\section{REFERENCES}

[1] B. Gharnali and S. Alipour, "MRI Image Segmentation Using Conditional Spatial FCM Based on Kernel-Induced Distance Measure," Engineering, Technology \& Applied Science Research, vol. 8, no. 3, pp. 2985-2990, Jun. 2018, https://doi.org/10.48084/etasr.1999.

[2] L. Jordanova, L. Laskov, and D. Dobrev, "Influence of BCH and LDPC Code Parameters on the BER Characteristic of Satellite DVB Channels," Engineering, Technology \& Applied Science Research, vol. 4, no. 1, pp. 591-595, Feb. 2014, https://doi.org/10.48084/etasr.394.

[3] C. E. Shannon, "A Mathematical Theory of Communication," The Bell System Technical Journal, vol. 27, pp. 379-423, 623-656, Jul., Oct. 1948.

[4] C. Berrou, A. Glavieux, and P. Thitimajshima, "Near Shannon limit error-correcting coding and decoding: Turbo-codes. 1," in Proceedings of ICC '93 - IEEE International Conference on Communications, Geneva, Switzerland, May 1993, vol. 2, pp. 1064-1070, https://doi.org/ 10.1109/ICC.1993.397441

[5] Ha-Young Yang, Suk-Hyun Yoon, and Chang-Con Kang, "Iterative decoding of serially concatenated convolutional codes applying the SOVA," in VTC '98. 48th IEEE Vehicular Technology Conference. Pathway to Global Wireless Revolution (Cat. No.98CH36151), Ottawa, Canada, May 1998, vol. 1, pp. 353-357, https://doi.org/10.1109/ VETEC.1998.686594.

[6] C. Berrou, Ed., Codes and turbo codes. Paris, France: Springer-Verlag, 2010.

[7] L. Mostari, R. Méliani, and A. Bounoua, "Turbo-code in a bit inter leaved tubo-coded modulation system," in CMT'2010, Ecole Supérieure de Technologie, Hassan 2 University, Casablanca, Morocco, 2010.

[8] "In pictures ... this is how the Corona virus appears in the infected lungs," Ramallah News, Mar. 14, 2020. https://ramallah.news/post/ 151117/ (accessed Feb. 24, 2021).

[9] M. Fayssal, "Optimisation d'une transmission d'images fixes utilisant un turbo-code," Ph.D. dissertation, University of Djillali Liabes, Sidi bel Abbes, Algeria, 2019.

[10] P. Robertson and T. Wörz, "Coded modulation scheme employing turbo codes," Electronics Letters, vol. 31, no. 18, pp. 1546-1547, Aug. 1995, https://doi.org/10.1049/el:19951064.

[11] P. Hoeher, "New Iterative ('Turbo') Decoding Algorithms," in in Proc. International Symposium on Turbo Codes \& Related Topics, Brest, France, 1997, pp. 63-70.

[12] J. D. Kene and K. D. Kulat, "Soft Output Decoding Algorithm for Turbo Codes Implementation in Mobile Wi-Max Environment," Procedia Technology, vol. 6, pp. 666-673, 2012, https://doi.org/10.1016/ j.protcy.2012.10.080.

[13] P. Robertson and T. Worz, "Bandwidth-efficient turbo trellis-coded modulation using punctured component codes," IEEE Journal on Selected Areas in Communications, vol. 16, no. 2, pp. 206-218, Feb. 1998, https://doi.org/10.1109/49.661109.

[14] G. Ungerboeck, "Channel coding with multilevel/phase signals," IEEE Transactions on Information Theory, vol. 28, no. 1, pp. 55-67, Jan. 1982, https://doi.org/10.1109/TIT.1982.1056454.

[15] M. R. Lahcene, "Amelioration des performances des codeur/decodeur sur canal WIMAX," Ph.D. dissertation, University of Tahri Mohammed, Bechar, Algeria, 2018.

[16] M. R. Lahcene, A. Bassou, M. Beladgham, and A. Taleb-Ahmed, "On the Employment of Unpunctured Turbo-Trellis Coded Modulation Channel Coding to Improve the Performance of WiMax System," International Journal on Communications Antenna and Propagation, vol. 8, no. 3, pp. 248-256-256, Jun. 2018, https://doi.org/10.15866/ irecap.v8i3.13609.

[17] F. Menezla, M. S. Bendelhoum, Z. Mahdjoub, L. M. Reda, and R. Meliani, "The Effect of Error Correcting Codes in the Chain of Transmission and Comparison between the Performances of these Codes," in 2019 6th International Conference on Image and Signal Processing and their Applications (ISPA), Mostaganem, Algeria, Nov. 2019, https://doi.org/10.1109/ISPA48434.2019.8966917.
[18] H. Mounira, "Reception dans un systeme d'acces multibles a repartition par code et application aux mode FDD et TDD de l'UMTS," Ph.D. dissertation, University of Biskra, Biskra, Algeria, 2014.

[19] K. D. Eduardo, "Les code correcteurs d'erreurs LDPC," M.S. thesis, University of Sidi bel Abbes, Sidi bel Abbes, Algeria, 2012.

[20] F. Menezla, R. Meliani, and Z. Mahdjoub, "Compartive study on performance of the LDPC codes and turbo-codes used in the image transmission in the gaussian and rayleigh channel," Journal of Applied Research and Technology, vol. 17, no. 2, Oct. 2019, https://doi.org/ 10.22201/icat.16656423.2019.17.2.802.

[21] B. Mohamed, B. Abdesselam, B. Mohammed, T.-A. Abdelmalik, and M. L. Abdelmounaim, "International Journal of Image, Graphics and Signal Processing(IJIGSP)," International Journal of Image, Graphics and Signal Processing(IJIGSP), vol. 6, no. 10, pp. 10-17, Sep. 2014.

[22] S. Wang, C. Perrine, C. Olivier, and C. Chatellier, "Stratégie de codage conjoint pour la transmission de séquences d'images via un canal bruité," Nov. 2007, Accessed: Feb. 24, 2021. [Online]. Available: https://hal.archives-ouvertes.fr/hal-00348734. 